\title{
The Costs of Implementing the Majority Principle: The Golden Voting Rule
}

By

\author{
Eyal Baharad ${ }^{*}$ and Shmuel Nitzan ${ }^{+}$
}

\begin{abstract}
In a context of constitutional choice of a voting rule, this paper presents an economic analysis of scoring rules that identifies the golden voting rule under the impartial culture assumption. This golden rule depends on the weights $\beta$ and (1- $\beta$ ) assigned to two types of costs: the cost of majority decisiveness ('tyranny') and the cost of the 'erosion' in the majority principle. Our first main result establishes that in voting contexts where the number of voters $n$ is typically considerably larger than the number of candidates $k$, the golden voting rule is the inverse plurality rule for almost any positive $\beta$. Irrespective of $n$ and $k$, the golden voting rule is the inverse plurality rule if $\beta \unrhd 1 / 2$. This hitherto almost unnoticed rule outperforms any other scoring rule in eliminating majority decisiveness. The golden voting rule is, however, the plurality rule, the most widely used voting rule that does not allow even the slightest 'erosion' in the majority principle, when $\beta=0$. Our second main result establishes that for sufficiently "small size" voting bodies, the set of potential golden rules consists at most of just three rules: the plurality rule, the Borda rule and the inverse plurality rule. On the one hand, this finding provides a new rationalization to the central role the former two rules play in practice and in the voting theory literature. On the other hand, it provides further support to the inverse plurality rule; not only that it is the golden rule in voting contexts, it also belongs, together with the plurality rule and the Borda method of counts, to the "exclusive" set of potential golden voting rules in small committees.
\end{abstract}

\footnotetext{
- We are indebted to Jim Buchanan, Amichai Glazer, Noa Nitzan and Ken Shepsle for their useful comments.

* Department of Economics, The University of Haifa, Haifa 31905, Israel. E-mail: baharad@econ.haifa.ac.il

+ Department of Economics, Bar Ilan University, Ramat Gan 52900, Israel. E-mail: nitzans@ mail.biu.ac.il
} 


\section{Introduction}

The framework proposed by Buchanan and Tullock (1962) to study the constitutional choice of a voting rule focused on the class of special-majority rules that are applied in the dichotomous setting of passing or failing to pass an issue. Their proposed normative criterion was the minimization of the sum of external costs and decisionmaking costs. This general approach was applied by Rae (1969) who illustrated that under certain symmetry assumptions simple majority rule emerges as the optimal rule; the rule that minimizes the probability that a representative voter supports an issue that is defeated or opposes an issue that wins. A formal proof of this result was provided by Taylor (1969).

Within the very different uncertain dichotomous choice setting, where individual preferences are assumed to be identical, yet, decisional skills may differ, Nitzan and Paroush (1982), and, more recently, Ben-Yashar and Nitzan (1997) have shown that some weighted qualified majority rule is always the optimal decisionmaking rule; the rule that maximizes the expected payoff of the group members. In particular, this optimal rule can be the simple majority rule, the expert rule, or any qualified majority rule. ${ }^{1}$

In a multi-candidate context, there have been no attempts to apply Buchanan and Tullock (1962) 'calculus of consent' approach. Nevertheless, an attempt was made to identify the optimal voting rule within the class of scoring rules (that are also referred to as positional rules or point-voting schemes). In particular, it has been shown that the Borda method of counts maximizes the average ranking of the selected candidate or, equivalently, the candidate chosen by this rule has the largest number of supporters in binary comparisons with other candidates, Austen-Smith and Banks (1999), Farkas and Nitzan (1979), Saari (1990), (1995), (2000). No wonder then that Merrill (1984) and Bordley (1983) presented simulation results showing that the Borda rule achieves a higher aggregate utility level relative to some alternative voting rules (the plurality rule, the runoff election method and approval voting).

\footnotetext{
${ }^{1}$ On the question of how well voting mechanisms aggregate private information and preferences in a strategic dichotomous setting, see Dekel and Piccione (2000) and Li, Rosen and Suen (2001) and references therein.
} 
The appeal of scoring rules stems from their unbiasedness toward voters (scoring rules are anonymous) and toward alternatives (scoring rules are neutral) ${ }^{2}$ while allowing some limited uniform expression of preference intensities. As recently shown by Baharad and Nitzan (2002), these properties enable scoring rules to eliminate the decisiveness ('tyranny') of certain majorities, even under coordinated strategic voting. Their main result calls attention to the possible amelioration of majority decisiveness by scoring rules. However, it implies that, in general, elimination of such decisiveness is impossible. That is, any particular scoring rule is still vulnerable to the tyranny of any majority that is equal to or is larger than some fraction $\alpha^{+}$of the number of the voters. The use of a scoring rule therefore entails a certain restriction on the ability of the minority to effectively express its preference intensities. No attempt is made in Baharad and Nitzan (2002) to measure the severity of this restriction. Furthermore, while focusing on the prevention of majority tyranny, the authors completely ignore the implications that the application of a particular scoring rule has in terms of stultifying the will of the majority.

The objective of the current paper is to take into account the severity of both aspects of scoring rules and present a new application of the constitutional approach proposed by Buchanan and Tullock for identifying the optimal voting rule in a multicandidate setting. In other words, our main purpose is to identify the rule that provides the golden compromise or balance between effectively implementing the will of a majority and preventing it from being tyrannical. The identification of this rule is important not only in theory, but also in practice, given the continuing controversy over different election methods. ${ }^{3}$

To clarify the nature of the normative question on which we focus, notice that, on the one hand, the tyranny of any $\alpha$-majority, $\alpha \geq \alpha^{+}$, corresponding to a particular scoring rule implies one type of costs that can be measured by the probability that some such $\alpha$-majority tyranny is realized. On the other hand, the effectivity of that scoring rule to eliminate the tyranny of any $\alpha$-majority, $\alpha<\alpha^{+}$, can be considered as a violation of the majority principle. The 'erosion' of this principle is a second type of

\footnotetext{
${ }^{2}$ Anonymity requires invariance of the voting rule with respect to permutations of voters' preferences. Neutrality requires appropriate variance of the voting rule with respect to permutations of the alternatives; if the alternatives are permuted in the preferences of the voters, then the alternative/s selected by the voting rule change accordingly.

${ }^{3}$ E.g., the role of the Electoral College in presidential elections in the US.
} 
costs associated with the application of a scoring rule. This cost can be measured by the probability that a selection of a candidate that is unanimously preferred by the majority is not guaranteed. The computation of these two types of costs is based on the standard impartial-culture assumption, namely, that all possible preference profiles are equally probable. The two types of probabilistic costs $C^{1}(\cdot)$ and $C^{2}(\cdot)$ mentioned above depend on the particular $\alpha^{+}$corresponding to a scoring rule, on the number of alternatives $k$ and on the number of voters $n$. The normative constitutional motive to identify the golden voting rule takes into account the two types of costs of implementing the majority principle. That is, it can be based on some function that is positively related to the costs $C^{1}(\cdot)$ and $C^{2}(\cdot)$. A natural such function is the weighted sum $\beta C^{1}(\cdot)+(1-\beta) C^{2}(\cdot)$, where $\beta$ and $(1-\beta)$ are the weights assigned to the two types of costs. ${ }^{4}$ The golden voting rule is a scoring rule that minimizes this weighted sum. This scoring rule belongs to the equivalence class of scoring rules corresponding to some $\alpha^{*}$. In other words, the optimal scoring rule is characterized by the golden degree of majority tyranny $\alpha^{*}$. The conditions that characterize the golden voting rule are stated in terms of $\beta, k$ and $n$. Using these conditions, the main objective of this paper is to identify the golden voting rule and, in particular, study the circumstances that give rise to the emergence of the plurality rule, the Borda rule and the inverse plurality rule as the golden voting rule. The latter hitherto unnoticed voting rule outperforms any other scoring rule in enabling the minority to effectively express its preference intensities or, alternatively, in eliminating the decisiveness of the majority.

Our first main result establishes that in typical voting contexts, where the number of voters $n$ is considerably larger than the number of candidates $k$, the golden voting rule is almost always the inverse plurality rule. In economic situations, where the number of alternatives often exceeds the number of decision makers, the inverse plurality rule is the golden voting rule if $\beta \geq 1 / 2$. Our second result provides further support to the inverse plurality rule by proving that in sufficiently small voting bodies (committees), this rule and the widely used and intensively studied plurality and Borda rules are the only scoring rules that are potential golden voting rules.

\footnotetext{
${ }^{4}$ In section 4 we discuss the robustness of our results with respect to two types of changes in the objective function.
} 
In the next section we introduce our framework and some general properties of voting rules and, in particular, of scoring rules. Section 3 is devoted to the two types of 'costs" associated with the implementation of the majority principle by any scoring rule. Section 4 contains the analysis of the 'golden voting rule'. The study of potential golden rules in small voting bodies and the comparison between the conditions that justify the selection of these rules is contained in Section 5. The last section contains a brief summary and concluding remarks.

\section{The framework}

Let $N=\{1, \ldots, n\}, n \geq 3$, denote a finite set of voters and $A$ a finite set consisting of $k$ distinct alternatives, $k \geq 3$. Individual preference relations are defined over $A$ and are assumed to be strict (indifference is not allowed).

In this study we focus on voting rules that are usually referred to as scoring rules $^{5}$. A voting rule specifies for any given preference profile a non empty set of alternatives in $A$. Unbiasedness, which is a basic property of the voting rules on which we focus has two aspects ${ }^{6}$ :

(i) Unbiasedness toward voters, known as anonymity, requires invariance of the voting rule with respect to permutations of voters' preferences; if the preference relations of the voters are permuted, then the outcome of the voting rule is not affected. Put differently, the voting outcome is independent of the names of the voters.

(ii) Unbiasedness toward alternatives, which is referred to as neutrality, requires appropriate variance of the voting rule with respect to permutations of the alternatives in $A$; if the alternatives are permuted in the preferences of the voters on $A$, then the alternative/s selected by the voting rule change accordingly. In other words, the voting outcome is independent of the names of the candidates.

Let $S=\left\{S_{1}, S_{2}, \ldots, S_{k}\right\}$ be a monotone sequence of real numbers, $S_{1} \leq S_{2} \leq \ldots \leq S_{k}$ and $S_{1}<S_{k}$. Each of the $n$ voters ranks the candidates assigning $S_{i}$ points to the candidate ranked $k+1-i$. That is, each voter assigns $S_{1}$ points to the one ranked last, $S_{2}$

\footnotetext{
${ }^{5}$ In the literature scoring rules are sometimes referred to as positional rules, Gardenfors (1973) or as point-voting schemes, Mueller (2003).

${ }^{6}$ The two other conditions that together with the unbiasedness condition uniquely characterize scoring rules are reinforcement and continuity, see Young (1975) and Brams and Fishburn (2002). For a
} 
points to the one ranked next to the last, and so on. A scoring rule selects the candidates that receive the maximal total score.

Scoring rules allow a limited uniform degree of intra-personal preference intensity because scores are assigned to the alternatives according to their ranking. The intensity of preferring one alternative to another one can be represented by the difference in their scores. The two most well known scoring rules are the plurality and the Borda rules ${ }^{7}$.

Under the plurality rule, $S^{\mathrm{p}}=\left\{S_{1}, S_{2}, \ldots, S_{k}\right\}=\{0, \ldots, 0,1\}$. That is, the candidate who is ranked first by the largest number of voters is elected. The plurality rule is the most commonly used scoring rule. ${ }^{8}$

Under the Borda rule, $S^{\mathrm{B}}=\left\{S_{1}, S_{2}, \ldots, S_{k-1}, S_{k}\right\}=\{0,1, \ldots, k-2, k-1\} .9$

This study draws attention to the inverse plurality rule, a scoring rule that hitherto has not attracted almost any attention.

Under the inverse plurality rule, $S^{\mathrm{ip}}=\left\{S_{1}, S_{2}, \ldots, S_{k}\right\}=\{0,1, \ldots, 1\}$. The informational requirements of the plurality rule and of the inverse plurality rule are very modest. In the former case an individual has to report just his most preferred alternative. In the latter case the application of the rule is possible when every individual reports just his worst alternative $\mathrm{e}^{10}$. The unique properties of this scoring rule and its special appeal in voting contexts are discussed in the sequel.

\section{The costs of implementing the majority principle}

\subsection{Majority decisiveness}

Focusing on the extent of majority decisiveness allowed by scoring rules, we define $\alpha$-majority decisiveness $(1 / 2<\alpha \leq 1$ and $\alpha$ being a fraction with a denominator

comprehensive survey of alternative axiomatic characterizations of scoring rules see Chebotarev and Shamis (1998).

7 'Approval voting', Brams and Fishburn (1978), and the 'unconstrained point-voting scheme', Mueller (2003), are two related voting rules. Both of these rules can be considered as variants of a scoring rule because they are individual specific, 'flexible scoring rules'. Under the unconstrained point-voting scheme, often referred to as cumulative voting, each voter has complete flexibility in allocating his initial endowment of points. The initial point endowments of the voters are equal and an elected candidate is one that accumulates a maximal number of points.

${ }^{8}$ For an axiomatic characterization of the plurality rule, see Richelson (1978).

${ }^{9}$ For axiomatic characterizations of the Borda rule, see Nitzan and Rubinstein (1981), Saari (1990), Young (1974). For a discussion on the Condorcet consistency of this rule, see Baharad and Nitzan (2003). 
$n)$ as the ability of any majority group of size $\alpha$ to impose its will whenever its members share a common view regarding the desirable collective decision ${ }^{11}$. Such an ability implies that the corresponding (1- $\alpha$ ) minority group cannot express its preference intensity in a minimally effective way; there does not exist even a single profile under which the minority group has a veto power. In our unbiased voting context the decisiveness of the majority is therefore referred to as majority tyranny. Let $f(t)$ be the maximal fraction with a denominator $n$ that is smaller than or equal to $\left(t+\frac{1}{n}\right)^{12}$. The following lemma specifies the minimal degrees of decisiveness corresponding to the plurality rule, $\alpha^{+}\left(S^{\mathrm{p}}\right)=\alpha^{\mathrm{p}}$, the Borda rule, $\alpha^{+}\left(S^{\mathrm{B}}\right)=\alpha^{\mathrm{B}}$, and the inverse plurality rule, $\alpha^{+}\left(S^{\mathrm{ip}}\right)=\alpha^{\mathrm{ip}}$.

Lemma 1: $\quad$ (i) $\alpha^{+}\left(S^{\mathrm{p}}\right)=\alpha^{\mathrm{p}}=f(1 / 2)$

$$
\begin{aligned}
& \text { (ii) } \alpha^{+}\left(S^{\mathrm{B}}\right)=\alpha^{\mathrm{B}}=f\left(\frac{2 k-2}{3 k-2}\right) \\
& \text { (iii) } \alpha^{+}\left(S^{\mathrm{ip}}\right)=\alpha^{\mathrm{ip}}=f\left(\frac{k-1}{k}\right)
\end{aligned}
$$

Proof: See appendix.

The plurality rule is an extreme scoring rule that allows the decisiveness of any majority. The Borda rule is an intermediate rule. It can be easily verified that, for a sufficiently large number of alternatives $k, \alpha^{+}\left(S^{\mathrm{B}}\right)=f\left(\frac{2}{3}\right)$. The inverse plurality rule is another extreme scoring rule. This rule provides the highest minimal degree of decisiveness, that is, $\operatorname{argmax} \alpha^{+}(S)=\alpha^{+}\left(S^{\mathrm{ip}}\right)=f\left(\frac{k-1}{k}\right)$. Notice that when $k \geq n$ this rule eliminates any $\alpha$-majority decisiveness, $1 / 2<\alpha<1$. The inverse plurality rule can

\footnotetext{
${ }^{10}$ In Saari (1995) this rule is referred to as the anti-plurality rule. In Myerson (2002) this rule is called negative voting.

${ }^{11}$ We assume that the majority-coalition members resort to coordinated strategic voting. Coordinated voting requires that the majority members assign the largest number of scores to their preferred alternative and that they spread their remaining scores uniformly over the other alternatives. Such coordination facilitates the attainment of decisiveness. That is, it increases the strength of the majority to impose the selection of their preferred alternative regardless of the votes of the minority.

12 Notice that whether $\alpha^{*}(S)$ is or is not a fraction with a denominator $n$, an $f\left(\alpha^{*}(S)\right)$-majority coalition can impose the selection of its unanimously favored alternative in the strict sense, that is, the $\left(1-\alpha^{*}(S)\right)$-minority group cannot also secure the selection of some other alternative.
} 
therefore be considered as the "unanimity rule analogue". The analogy between this scoring rule and the unanimity rule ${ }^{13}$ is based on the property of every voter having a veto power under at least one preference profile. In other words, even a majority of $n$ 1 voters cannot always guarantee the selection of its most favorable candidate.

\subsection{Erosion of the majority principle}

On the one hand, when a scoring rule is vulnerable to the tyranny of any majority that is equal to or larger than $\alpha^{+}$, its use entails a certain restriction on the ability of the minority to effectively express its preference intensities. On the other hand, the ability of the scoring rule to effectively eliminate the tyranny of any majority that is smaller than $\alpha^{+}$can be considered as erosion of the majority principle (any such majority looses its perfect ability to effectively determine the outcome of the choice process). This erosion in the majority status and the tyranny implied by majority decisiveness are the two possible worrying aspects associated with the implementation of the majority principle and, in particular, with the use of scoring rules that entail different degrees of implementation of that principle represented by $\alpha^{+}-$the minimal degree of decisiveness.

\subsection{The costs}

To measure the costs of majority decisiveness, we make the standard assumption that all possible preference profiles are equally likely (the so called impartial culture assumption). The first type of costs of majority decisiveness corresponding to some $\alpha^{+}$is measured by $C^{1}\left(\alpha^{+}\right)$- the proportion of preference profiles in which an $\alpha-$ majority tyranny, $\alpha \geq \alpha^{+}$, is realized. The second type of costs of majority decisiveness corresponding to some $\alpha^{+}$is measured by $C^{2}\left(\alpha^{+}\right)$- the proportion of preference profiles in which the $\alpha$-majority principle, $\alpha<\alpha^{+}$, is eroded. Under the impartial culture assumption, ${ }^{14} C^{1}\left(\alpha^{+}\right)$is thus the probability that an $\alpha^{+}$- majority group, $\alpha \geq \alpha^{+}$, shares the same view regarding the most preferred alternative. $C^{2}\left(\alpha^{+}\right)$is the probability that an $\alpha$ - majority group, $\alpha<\alpha^{+}$, and the corresponding (1- $\left.\alpha\right)$-minority

\footnotetext{
${ }^{13}$ The unanimity rule was strongly advocated by Wicksell (1896) and Buchanan and Tullock (1962).

${ }^{14}$ For the sake of simplicity we base the formal analysis on the commonly used impartial culture assumption. Our results are robust, however, because they are valid under many alternative preference cultures.
} 
group unanimously prefer two different alternatives, and the majority cannot guarantee the selection of its most favorable alternative. In the Appendix we show that:

(1) $C^{1}\left(\alpha^{+}\right)=k \sum_{i=\alpha^{+} n}^{n-1}\left(\frac{1}{k}\right)^{i} \cdot\left(\frac{k-1}{k}\right)^{n-i} \cdot\left(\begin{array}{l}n \\ i\end{array}\right)$

and

(2) $C^{2}\left(\alpha^{+}\right)=\sum_{i=\lfloor 0.5 n+1}^{\alpha^{+} n-1}\left(\frac{1}{k}\right)^{i} \cdot\left(\frac{1}{k}\right)^{n-i} \cdot\left(\begin{array}{l}n \\ i\end{array}\right) \cdot\left(\frac{k !}{(k-2) !}\right)$

where $\lfloor t\rfloor$ is the largest integer that is equal to or smaller than $t$ and $\lceil t\rceil$ is the smallest integer that is equal to or larger than $t$.

\section{The golden voting rule}

Given the weight $\beta$ assigned to $C^{1}\left(\alpha^{+}\right)$, the number of alternatives $k$ and the number of voters $n$, the golden voting rule is a scoring rule that results in a minimal degree of decisiveness $\alpha^{+}$that solves the following problem:

$$
\underset{\alpha^{+}}{\operatorname{Min} \beta \cdot C^{1}\left(\alpha^{+}\right)+(1-\beta) \cdot C^{2}\left(\alpha^{+}\right)}
$$

or,

$$
\begin{aligned}
& \operatorname{Min}^{+} \beta \cdot k \sum_{i=\alpha^{+} n}^{n-1}\left(\frac{1}{k}\right)^{i} \cdot\left(\frac{k-1}{k}\right)^{n-i} \cdot\left(\begin{array}{l}
n \\
i
\end{array}\right)+ \\
& (1-\beta) \cdot \sum_{i=[0.5 n+1}^{\alpha^{+} n-1}\left(\frac{1}{k}\right)^{i} \cdot\left(\frac{1}{k}\right)^{n-i} \cdot\left(\begin{array}{l}
n \\
i
\end{array}\right) \cdot\left(\frac{k !}{(k-2) !}\right)
\end{aligned}
$$


Let $\Delta C_{-}^{i}(\alpha)=C^{i}(\alpha)-C^{i}\left(\alpha-\frac{1}{n}\right)$ and $\Delta C_{+}^{i}(\alpha)=C^{i}\left(\alpha+\frac{1}{n}\right)-C^{i}(\alpha)$, for $i=1,2$.

The necessary and sufficient conditions for an interior solution $\alpha^{*}$ of the above problem are:

$$
\beta \Delta C_{-}^{1}\left(\alpha^{*}\right)+(1-\beta) \Delta C_{-}^{2}\left(\alpha^{*}\right) \leq 0 \quad \text { and } \quad \beta \Delta C_{+}^{1}\left(\alpha^{*}\right)+(1-\beta) \Delta C_{+}^{2}\left(\alpha^{*}\right) \geq 0
$$

or, at $\alpha^{*}$,

$$
\frac{\Delta C_{-}^{2}}{\Delta C_{-}^{2}-\Delta C_{-}^{1}} \leq \beta \leq \frac{\Delta C_{+}^{2}}{\Delta C_{+}^{2}-\Delta C_{+}^{1}}
$$

By substituting (1) and (2), the necessary and sufficient conditions are:

$$
\frac{1}{1+(k-1)^{\left(1-\alpha^{*}\right) n}} \leq \beta \leq \frac{1}{1+(k-1)\left(1-\alpha^{*}\right) n-1}
$$

Notice that when $\alpha^{*}$ is a corner solution only one of the above inequalities holds. That is, when $\alpha^{*}=f(1 / 2)$, the necessary and sufficient condition is:

$$
\beta \leq \frac{1}{1+(k-1)^{\left(1-\alpha^{*}\right) n-1}}
$$

When $\alpha^{*}=f\left(\frac{k-1}{k}\right)$, the necessary and sufficient condition is:

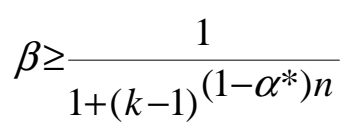

By the above conditions and Lemma 1 we get the following result: 


\section{Theorem 1:}

(i) When $n \leq k$, the inverse plurality rule is the golden voting rule if $1 / 2 \leq \beta \leq 1$.

(ii) When $n>k$ and $n$ is sufficiently large, the inverse plurality rule is the golden voting rule for every $\beta, \delta \leq \beta \leq 1, \delta \rightarrow 0$.

(iii) Independent of $n$ and $k$, if $\beta=0$, the plurality rule is the golden voting rule.

Proof: See appendix.

By part (i) of the theorem, when the number of alternatives exceeds the number of voters, the inverse plurality rule is the golden rule whenever the weight assigned to the cost of majority tyranny is equal to or larger than the weight assigned to the cost of the erosion in the majority principle. The intuition behind this result is the following: In such a case, even a majority of $n$-1 voters cannot guarantee the selection of its most favorable alternative, because there exists at least one alternative the score of which is equal to the score of the majority's preferred alternative or to that score minus one. The single-voter minority can thus assign zero points to this alternative, which prevents the possibility of it being the unique selection. Obviously, under the inverse plurality rule then, when $k \geq n$, the cost of majority tyranny is minimal. Since for given $n$ and $k, C^{1}(\cdot)>C^{2}(\cdot), \quad 1 / 2 \leq \beta \leq 1$ implies that the sum of the weighted costs is minimal. By part (ii) of the theorem, in a typical voting context where $n$ is sufficiently large, the inverse plurality rule is the golden voting rule for almost any positive $\beta$. This result is due to the existence of a very large gap between $C^{1}(\cdot)$ and $C^{2}(\cdot)$ when $n$ is large. In such a case even a very small $\beta$, the weight assigned to $C^{1}(\cdot)$, is sufficient to make $\beta \cdot C^{1}\left(\alpha^{+}\right)$the dominant term in (3). The minimization of the weighted sum in (3) therefore requires the minimization of $\beta \cdot C^{1}\left(\alpha^{+}\right)$, and, in turn, of $C^{1}(\cdot)$, which is attained by $\alpha^{\mathrm{ip}}$. In other words, the inverse plurality rule is the golden rule. By part (iii) of the theorem, the plurality rule is always the golden rule when $\beta=0$. Simply, when $\beta=0$, the objective function in (3) reduces to $C^{2}(\cdot)$. In such a case the golden voting rule minimizes the costs of the second type, that is, the costs of eroding the majority status. The plurality rule is the golden voting rule because under it $C^{2}(\cdot)=0$, the majority status is never eroded (a majority of any size larger than $1 / 2$ can always guarantee the selection of its favorable alternative). Parts (ii) and (iii) of the theorem therefore imply that in voting situations the Borda rule and, in fact, any 
other scoring rule, cannot be rationalized as a golden voting rule because the weight giving rise to the optimality of any such intermediate rule must be strictly positive and sufficiently close to 0 .

In Rae's (1969) and Taylor's (1969) binary voting setting, assigning equal weight to their costs (the probability that the representative voter's favored alternative is rejected and the probability that an alternative he opposes is selected) yields the unbiased simple majority rule as the optimal rule. Under different weights the optimal rules are qualified majority rules. These rules are biased; they violate the neutrality condition. In contrast, in the extended setting of our study, any scoring rule (recall that any such rule is unbiased) might be the golden voting rule. However, when the number of voters is large, the golden voting rule is almost always the inverse plurality rule. Let us conclude this section with two remarks on the robustness of the result. First, suppose that the normative criterion for comparing scoring rules is not represented by the weighted sum of the two types of probabilistic costs $C^{1}\left(\alpha^{+}\right)$and $C^{2}\left(\alpha^{+}\right)$, but rather by another function that is additive and monotone increasing in these costs. Since $C^{1}\left(\alpha^{+}\right)$is considerably larger than $C^{2}\left(\alpha^{+}\right)$, especially for a large $n$, under such a function we would get the same result. Second, suppose that the normative criterion for comparing scoring rules takes into account not only the probabilistic costs $C^{1}\left(\alpha^{+}\right)$and $C^{2}\left(\alpha^{+}\right)$, but also the complexity of the applied rules. In such a case the inverse plurality rule, like the plurality rule, is very simple to implement. Thus, taking into account the simplicity criterion would not change (and might even strengthen) the result.

\section{The potential golden rules in small voting bodies}

The set of distinct potential golden rules is represented by the set of distinct minimal degrees of decisiveness. The purpose of this section is to derive conditions for the set of potential golden rules to consist of just two or three distinct scoring rules. Since the set of distinct potential golden rules always contains the plurality rule and the inverse plurality rule, the necessary and sufficient condition for this set to consist of just these two rules is that the difference between $n f\left(\frac{k-1}{k}\right)$ and $n f\left(\frac{1}{2}\right)$, the largest and smallest minimal number of decisive voters corresponding, respectively, to the inverse plurality rule and to the plurality rule, is equal to 1 . Similarly, the necessary 
and sufficient condition for the set of potential golden rules to consist of just three voting rules is that the difference between $n f\left(\frac{k-1}{k}\right)$ and $n f\left(\frac{1}{2}\right)$ is equal to 2 . In general, the set of distinct potential golden rules consists of at most $m$ elements, $m \geq 2$, if and only if $n\left(\alpha^{\mathrm{ip}}-\alpha^{\mathrm{p}}\right) \leq m-1$. Focusing on the cases where $m=2$ and $m=3$ we obtain the following result:

\section{Theorem 2:}

(i) A necessary (a sufficient) condition for $m=2$, where $\alpha^{*} \in\left\{\alpha^{\mathrm{p}}, \alpha^{\mathrm{p}}\right\}$, is:

$$
n \leq\left\lfloor\frac{4 k-2}{k-2}\right\rfloor \quad\left(n \leq\left\lfloor\frac{k}{k-2}\right\rfloor\right)
$$

(ii) A necessary (a sufficient) condition for $m=3$ is:

$$
n \leq\left\lfloor\frac{6 k-2}{k-2}\right\rfloor \quad\left(n \leq\left\lfloor\frac{3 k}{k-2}\right\rfloor\right)
$$

Proof: See appendix.

The first (second) inequality in (10) is a necessary (a sufficient) condition for the set of potential golden voting rules to consist of the plurality rule and the inverse plurality rule. The maximal number of voters that satisfies the necessary condition weakly decreases with $k$ ranging between 10 (when $k=3$ ) and 4 (when $k$ is sufficiently large).

Table 1 illustrates the number of voters corresponding to $k=3,4,5,6$ that satisfy this condition. The condition is satisfied for ( $k=3$ and $n \leq 10, n \neq 9),(k=4$ and $n \leq 6)$, ( $k=5$ and $n \leq 6$ ), and ( $k=6$ and $n \leq 4)$. In these cases the Borda rule is equivalent to one of the two other rules, that is, $\alpha^{\mathrm{B}}=\alpha^{\mathrm{p}}$ or $\alpha^{\mathrm{B}}=\alpha^{\mathrm{ip}}$. 
Table 1: The potential golden voting rules when ( $k=3$ and $n \leq 14),(k=4$ and $n \leq 10)$, ( $k=5$ and $n \leq 8)$, and $(k=6$ and $n \leq 8)$, and the range of $\beta$, [min $\beta$, max $\beta$, under which the plurality, Borda and inverse plurality rules are the golden voting rules.

\begin{tabular}{|r|r|r|r|r|r|r|r|r|r|r|r|}
\hline & & \multicolumn{3}{|r|}{ PLURALITY } & \multicolumn{3}{|r|}{ BORDA } & \multicolumn{3}{|r|}{ INVERSE } \\
\hline$k$ & \multicolumn{1}{r|r|r|r|r|r|r|r|r|r|r|r|r|r|r|r|r|}{} \\
\hline 3 & 3 & $2 / 3$ & 0 & 0.50 & $2 / 3$ & 0 & 0.50 & $3 / 3$ & 0.50 & 1 \\
\hline 3 & 4 & $3 / 4$ & 0 & 1 & $3 / 4$ & 0 & 1 & $3 / 4$ & 0 & 1 \\
\hline 3 & 5 & $3 / 5$ & 0 & 0.33 & $3 / 5$ & 0 & 0.33 & $4 / 5$ & 0.33 & 1 \\
\hline 3 & 6 & $4 / 6$ & 0 & 0.33 & $4 / 6$ & 0 & 0.33 & $5 / 6$ & 0.33 & 1 \\
\hline 3 & 7 & $4 / 7$ & 0 & 0.20 & $5 / 7$ & 0.20 & 1 & $5 / 7$ & 0.20 & 1 \\
\hline 3 & 8 & $5 / 8$ & 0 & 0.20 & $5 / 8$ & 0 & 0.20 & $6 / 8$ & 0.20 & 1 \\
\hline 3 & 9 & $5 / 9$ & 0 & 0.11 & $6 / 9$ & 0.11 & 0.20 & $7 / 9$ & 0.20 & 1 \\
\hline 3 & 10 & $6 / 10$ & 0 & 0.11 & $6 / 10$ & 0 & 0.11 & $7 / 10$ & 0.11 & 1 \\
\hline 3 & 11 & $6 / 11$ & 0 & 0.06 & $7 / 11$ & 0.06 & 0.11 & $8 / 11$ & 0.11 & 1 \\
\hline 3 & 12 & $7 / 12$ & 0 & 0.06 & $7 / 12$ & 0 & 0.06 & $9 / 12$ & 0.11 & 1 \\
\hline 3 & 13 & $7 / 13$ & 0 & 0.03 & $8 / 13$ & 0.03 & 0.06 & $9 / 13$ & 0.06 & 1 \\
\hline 3 & 14 & $8 / 14$ & 0 & 0.03 & $9 / 14$ & 0.03 & 0.06 & $10 / 14$ & 0.06 & 1 \\
\hline & & & & & & & & & & \\
\hline 4 & 3 & $2 / 3$ & 0 & 0.50 & $2 / 3$ & 0 & 0.50 & $3 / 3$ & 0.50 & 1 \\
\hline 4 & 4 & $3 / 4$ & 0 & 0.50 & $3 / 4$ & 0 & 0.50 & $4 / 4$ & 0.50 & 1 \\
\hline 4 & 5 & $3 / 5$ & 0 & 0.25 & $4 / 5$ & 0.25 & 1 & $4 / 5$ & 0.25 & 1 \\
\hline 4 & 6 & $4 / 6$ & 0 & 0.25 & $4 / 6$ & 0 & 0.25 & $5 / 6$ & 0.25 & 1 \\
\hline 4 & 7 & $4 / 7$ & 0 & 0.10 & $5 / 7$ & 0.10 & 0.25 & $6 / 7$ & 0.25 & 1 \\
\hline 4 & 8 & $5 / 8$ & 0 & 0.10 & $5 / 8$ & 0 & 0.10 & $7 / 8$ & 0.25 & 1 \\
\hline 4 & 9 & $5 / 9$ & 0 & 0.04 & $6 / 9$ & 0.04 & 0.10 & $7 / 9$ & 0.10 & 1 \\
\hline 4 & 10 & $6 / 10$ & 0 & 0.04 & $7 / 10$ & 0.04 & 0.10 & $8 / 10$ & 0.10 & 1 \\
\hline & & & & & & & & & & \\
\hline 5 & 3 & $2 / 3$ & 0 & 0.50 & $2 / 3$ & 0 & 0.50 & $3 / 3$ & 0.50 & 1 \\
\hline 5 & 4 & $3 / 4$ & 0 & 0.50 & $3 / 4$ & 0 & 0.50 & $4 / 4$ & 0.50 & 1 \\
\hline 5 & 5 & $3 / 5$ & 0 & 0.20 & $4 / 5$ & 0.20 & 0.50 & $5 / 5$ & 0.50 & 1 \\
\hline 5 & 6 & $4 / 6$ & 0 & 0.20 & $4 / 6$ & 0 & 0.20 & $5 / 6$ & 0.20 & 1 \\
\hline 5 & 7 & $4 / 7$ & 0 & 0.06 & $5 / 7$ & 0.06 & 0.20 & $6 / 7$ & 0.20 & 1 \\
\hline 5 & 8 & $5 / 8$ & 0 & 0.06 & $5 / 8$ & 0 & 0.06 & $7 / 8$ & 0.20 & 1 \\
\hline & & & & & & & & & & \\
\hline 6 & 3 & $2 / 3$ & 0 & 0.50 & $2 / 3$ & 0 & 0.50 & $3 / 3$ & 0.50 & 1 \\
\hline 6 & 4 & $3 / 4$ & 0 & 0.50 & $3 / 4$ & 0 & 0.50 & $4 / 4$ & 0.50 & 1 \\
\hline 6 & 5 & $3 / 5$ & 0 & 0.17 & $4 / 5$ & 0.17 & 0.50 & $5 / 5$ & 0.50 & 1 \\
\hline 6 & 6 & $4 / 6$ & 0 & 0.17 & $4 / 6$ & 0 & 0.17 & $6 / 6$ & 0.50 & 1 \\
\hline 6 & 7 & $4 / 7$ & 0 & 0.04 & $5 / 7$ & 0.04 & 0.17 & $6 / 7$ & 0.17 & 1 \\
\hline 6 & 8 & $5 / 8$ & 0 & 0.04 & $6 / 8$ & 0.04 & 0.17 & $7 / 8$ & 0.17 & 1 \\
\hline
\end{tabular}


The first (second) inequality in (11) is a necessary (a sufficient) condition for the set of potential golden voting rules to consist of at most three voting rules. The maximal number of voters that satisfies the necessary condition weakly decreases with $k$ ranging between 16 (when $k=3$ ) and 6 (when $k$ is sufficiently large). This condition is satisfied for ( $k=3$ and $n \leq 14),(k=4$ and $n \leq 10),(k=5$ and $n \leq 8)$, and ( $k=6$ and $n \leq 8$ ). Notice that when $k=3$ the plurality rule, the Borda rule and the inverse plurality rule yield the three possible minimal degrees of decisiveness for any number of voters that is smaller than or equal to 14 , with the exception of $n=12$. When $(k=4$ and $n \leq 10),(k=5$ and $n \leq 7)$, and $(k=6$ and $n \leq 8)$ these three rules always yield the possible minimal degrees of decisiveness.

The table also specifies for any combination of $(k, n)$ the range of $\beta,[\mathrm{min} \beta$, $\max \beta$, under which the plurality, Borda and inverse plurality rules are the golden voting rules. For example, when $(k=3$ and $n=9)$ and $(k=4, n=9)$, the inverse plurality rule emerges as the golden voting rule for any $\beta$ that exceeds, respectively, 0.2 and 0.10 . Finally note that it can be shown that $(k=3$ and $n=4)$ is the necessary and sufficient condition for $m=1$, where $\alpha^{+}=\alpha^{i p}$.

\section{Conclusion}

This paper presents an economic approach for evaluating and, in turn, implementing the norm of respecting the majority principle. This approach, which is inspired by the one suggested forty years ago by Buchanan and Tullock (1962), is both operative and general. It is operative because it results in the identification of a golden voting rule, for any combination of the parameters $k, n$ and $\beta$. Furthermore, in voting situations this rule, the inverse plurality rule, is an especially easy to implement scoring rule. When $k \geq n$, this rule is the scoring-rule analogue of the unanimity rule. Our approach is general in two respects. First, we allow a voting setting where the number of alternatives is larger than two. Second, the ex-ante costs of decisiveness on which we focus vary across unbiased voting rules and not across biased and unbiased rules, as in the case of binary voting where the rules are the unbiased simple majority rule and the biased qualified majority rules. In the well known study of Rae (1969), which is based on 'political individualism', an equal weight is assigned to the two types of costs corresponding to the representative individual's two possible disappointments. In such 
a case, the optimal rule is the simple majority rule. When the weights assigned to the different costs are not equal, the optimal rule is a qualified majority rule. In the current study, which is based on 'political-economic idealism', viz., on the evaluation of the two possible 'evils' associated with majority decisiveness, when the weights assigned to the costs of majority tyranny and to the erosion of the majority principle are equal, the optimal rule is the inverse plurality rule. The same conclusion is valid in voting situations with a large number of voters, provided that the first type of costs is assigned almost any positive weight. In the extreme case, where the first type of costs is assigned a zero weight, the optimal rule is the commonly used plurality rule. In general, any scoring rule can be the optimal one. However, in typical voting situations the range of weights that give rise to an intermediate, non-extreme scoring rule like the Borda rule is extremely small. Qualitatively, these results are robust with respect to some changes in the functional form of the objective function that depends on the two type of probabilistic costs associated with the implementation of the majority principle. They are also robust with respect to a change in the objective function that takes into account the complexity of the rules, in addition to the two types of probabilistic costs.

Finally, the set of potential golden rules consists of at most two (at most three) rules in committees where the number of voters is small, $n \leq(4 k-2) /(k-2)$ $(n \leq(6 k-2) /(k-2))$. In the former case the plurality rule and the inverse plurality rule yield the two possible minimal degrees of decisiveness. In the latter case, with few exceptions, the Borda rule is the third rule that together with the plurality rule and the inverse plurality rule spans the range of the three possible minimal degrees of decisiveness. On the one hand, this finding provides a new rationalization to the central role the plurality rule and the Borda rule play in practice and in the voting theory literature. On the other hand, it provides further support to the inverse plurality rule; not only that it is the golden rule in voting contexts, it also belongs, together with the plurality rule and the Borda method of counts, to the "exclusive" set of potential golden voting rules in small committees. 


\section{References}

Austen-Smith, D. and J.S. Banks, (1999), Positive Political Theory I: Collective Preferences, Ann Arbor: University of Michigan Press.

Baharad, E. and S. Nitzan, (2002), "Ameliorating Majority Decisiveness Through Expression of Preference Intensity", American Political Science Review 96(4), 745-754.

Baharad, E. and S. Nitzan, (2003), "The Borda Rule, Condorcet Consistency and Condorcet Stability", Economic Theory, 22(3), 685-688.

Ben-Yashar, R. and S. Nitzan, (1997), “The Optimal Decision Rule for Fixed Size Committees in Dichotomous Choice Situations - The General Result”, International Economic Review, 38(1), 175-187.

Bordley, R. F., (1983), “A Pragmatic Method for Evaluating Election Schemes through Simulation”, American Political Science Review, 77, 123-141.

Brams, S.J. and P.C. Fishburn, (1978), “Approval Voting”, American Political Science Review, 72, 831-847.

Brams, S.J. and P.C. Fishburn (2002), "Voting Procedures", Chapter 4 in Arrow, K., Sen, A. and Suzumura, K. (eds), Handbook of Social Choice and Welfare, Amsterdam: Elsevier Science.

Buchanan, J. M. and G. Tullock, (1962), The Calculus of Consent, Ann Arbor: University of Michigan Press.

Chebotarev, P.U. and E. Shamis, (1998), "Characterizations of Scoring Methods for Preference Aggregation", Annals of Operations Research, 80, 299-332.

Dekel, E. and M. Piccione, (2000), "Sequential Voting Procedures in Symmetric Binary Elections”, Journal of Political Economy, 108, 3455.

Farkas, D. and S. Nitzan, (1979), "The Borda Rule and Pareto Stability: A Comment", Econometrica, 47, 1305-1306.

Gardenfors, P., (1973), "Positional Voting Functions", Theory and Decision, $4,1-24$.

Li, H., S. Rosen and W. Suen, (2001), "Conflicts and Common Interests in Committees", American Economic Review, 91, 1478-1497. 
Myerson, R.B., (2002), "Comparison of Scoring Rules in Poisson Voting Games”, Journal of Economic Theory, 103, 219-251.

Merrill, S. III., (1984), “A Comparison of Efficiency of Multicandidate Electoral Systems", American Political Science Review, 28, 23-48.

Mueller, D., (2003), Public Choice III, Cambridge, U.K.: Cambridge University Press.

Nitzan, S. and J. Paroush (1982), "Optimal Decision Rules in Uncertain Dichotomous Choice Situations", International Economic Review, 23, 289-297.

Nitzan, S. and A. Rubinstein, (1981), “A Further Characterization of Borda Ranking Method", Public Choice, 36, 153-8.

Rae, D. W., (1969), "Decision Rules and Individual Values in Constitutional Choice”, American Political Science Review, 63, 40-56.

Richelson, J.T., (1978), “A Characterization Result for the Plurality Rule”, Journal of Economic Theory, 19, 548-550.

Saari, D.G., (1990), “The Borda Dictionary”, Social Choice and Welfare, 7, 279-317.

Saari, D.G., (1995), Basic Geometry of Voting, Springer, Berlin.

Saari, D.G., (2000), "Mathematical Structure of Voting Paradoxes II: Positional Voting”, Economic Theory, 15(1), 55-102.

Saari, D.G. and K.K. Sieberg, (2001), "The Sum of the Parts Can Violate the Whole”, American Political Science Review, 95, 415-433.

Sen, A., (1970), Collective Choice and Social Welfare, Holden Day Inc., Cambridge.

Taylor, M J., (1969), "Proof of a Theorem on Majority Rule", Behavioral Science, 14, 228-231.

Wicksell, K. (1896), “A New Principle of Just Taxation”, Finanztheoretische Untersuchungen, Jena, reprinted in Musgrave, R. and Peacock, A.T., eds. Classics in the Theory of Public Finance, New York:McGraw Hill, 1980.

Young, H.P., (1974), “An Axiomatization of Borda's Rule”, Journal of Economic Theory, 9, 43-52.

Young, H.P., (1975), “Social Choice Scoring Functions”, SIAM Journal of Applied Math., 28, 824-838. 


\section{Appendix}

The decisiveness of a certain majority group is realized when all its members prefer the same candidate. The probability that such a majority group of size $i$ chooses, unanimously, the same candidate is $k\left(\frac{1}{k}\right)^{i}$. The probability that the corresponding minority group of size $n-i$ chooses any other candidate than the one chosen by the majority is $\left(\frac{k-1}{k}\right)^{n-i}$. Multiplying the product of the above probabilities by $\left(\begin{array}{l}n \\ i\end{array}\right)$ yields (due to the anonymity of the scoring rule) the probability that the decisiveness of some $i$-majority group is realized. Summing this term over all possible such $i$ - majority groups yields

(1) $C^{1}\left(\alpha^{+}\right)=k \sum_{i=\alpha^{+} n}^{n-1}\left(\frac{1}{k}\right)^{i} \cdot\left(\frac{k-1}{k}\right)^{n-i} \cdot\left(\begin{array}{l}n \\ i\end{array}\right)$

Notice that when $\alpha^{+}$is equal to 1 , the cost associated with majority tyranny is 0 . In such a case no terms are summed up in (1).

Similarly, letting $i$ denote the size of non-decisive majorities, $i$ ranging from $\lfloor 0.5 n+1\rfloor$, to $\alpha^{+} n-1, \quad\left(\frac{1}{k}\right)^{i}$ and $\left(\frac{1}{k}\right)^{n-i}$ are , respectively, the probabilities that a majority of size $i$ and a minority of size $n-i$ choose, unanimously, two different alternatives. The number of possible partitions of the voters to groups of size $i$ and $n-i$ is $\left(\begin{array}{l}n \\ i\end{array}\right)$. The number of pairs of different alternatives unanimously chosen by the majority and minority groups is $\left(\frac{k !}{(k-2) !}\right)$. Hence the probability of erosion of the majority principle is

(2) $C^{2}\left(\alpha^{+}\right)=\sum_{i=\lfloor 0.5 n+1}^{\alpha^{+} n-1}\left(\frac{1}{k}\right)^{i} \cdot\left(\frac{1}{k}\right)^{n-i} \cdot\left(\begin{array}{l}n \\ i\end{array}\right) \cdot\left(\frac{k !}{(k-2) !}\right)$ 
Notice than when $\alpha^{+}$is equal to $\lfloor 0.5 n+1\rfloor$, the cost associated with erosion in the majority status is 0 . In such a case no terms are summed up in (2).

Lemma 1: $\quad$ (i) $\alpha^{+}\left(S^{\mathrm{p}}\right)=\alpha^{\mathrm{p}}=f(1 / 2)$

$$
\begin{aligned}
& \text { (ii) } \alpha^{+}\left(S^{\mathrm{B}}\right)=\alpha^{\mathrm{B}}=f\left(\frac{2 k-2}{3 k-2}\right) \\
& \text { (iii) } \alpha^{+}\left(S^{\mathrm{ip}}\right)=\alpha^{\mathrm{ip}}=f\left(\frac{k-1}{k}\right)
\end{aligned}
$$

Proof: By the main result in Baharad and Nitzan (2002), under a scoring rule defined by $S=\left\{S_{1}, S_{2}, \ldots, S_{k}\right\}$ and perfectly coordinated voting, the minimal degree of decisiveness is equal to

$$
\alpha^{+}(S)=f\left(\frac{S_{k}-S_{1}}{2 S_{k}-S_{1}-\bar{S}}\right)
$$

where $\bar{S}=\sum_{i=1}^{k-1} S_{i} /(k-1)$. Substituting into (A1) the $S_{1}$ and $S_{k}$ corresponding to the plurality rule, the Borda rule and the inverse plurality rule, we directly obtain that the minimal degrees of decisiveness corresponding to these rules are equal, respectively, to $f(1 / 2), f\left(\frac{2 k-2}{3 k-2}\right)$ and $f\left(\frac{k-1}{k}\right)$.

\section{Theorem 1:}

(i) When $n \leq k$, the inverse plurality rule is the golden voting rule if $1 / 2 \leq \beta \leq 1$.

(ii) When $n>k$ and $n$ is sufficiently large, the inverse plurality rule is the golden voting rule for every $\beta, \delta \leq \beta \leq 1, \delta \rightarrow 0$.

(iii) Independent of $n$ and $k$, if $\beta=0$, the plurality rule is the golden voting rule.

\section{Proof:}

(i) By substituting $\alpha^{\text {ip }}$ (see Lemma 1 (iii)) into (9), we obtain that the golden voting rule is the inverse plurality rule if the weight $\beta^{\text {ip }}$ satisfies:

$$
\frac{1}{1+(k-1)^{\left\lfloor\frac{n}{k}\right\rfloor}} \leq \beta^{i p} \leq 1
$$

Since $n \leq k,\left\lfloor\frac{n}{k}\right\rfloor=0$ and, in turn, $1 / 2 \leq \beta^{\text {ip }} \leq 1$. 
(ii) By substituting $\alpha^{\mathrm{p}}$ (see Lemma 1 (iii)) into (9), we obtain that the golden voting rule is the inverse plurality rule if the weight $\beta^{\text {ip }}$ satisfies (A2).

Since $n>k>2, \lim _{n \rightarrow \infty} \frac{1}{1+(k-1)^{\left\lfloor\frac{n}{k}\right\rfloor}} \rightarrow 0$. That is, $\alpha^{\mathrm{p}}$ satisfies (9) and therefore it is the solution of problem (3) for almost every positive $\beta$.

(iii) By substituting $\alpha^{\mathrm{p}}$ (see Lemma 1(i)) into (8), we obtain that the golden voting rule is the plurality rule if the weight $\beta^{\mathrm{p}}$ satisfies:

$$
0 \leq \beta^{p} \leq \frac{1}{1+(k-1)^{\left[\frac{n}{2}\right]-1}}
$$

Obviously, $\beta=0$ satisfies this condition.

\section{Theorem 2:}

(i) A necessary (a sufficient) condition for $m=2$, where $\alpha^{*} \in\left\{\alpha^{\mathrm{p}}, \alpha^{\mathrm{p}}\right\}$, is:

$$
n \leq\left\lfloor\frac{4 k-2}{k-2}\right\rfloor \quad\left(n \leq\left\lfloor\frac{k}{k-2}\right\rfloor\right)
$$

(ii) A necessary (a sufficient) condition for $m=3$ is:

$$
n \leq\left\lfloor\frac{6 k-2}{k-2}\right\rfloor \quad\left(n \leq\left\lfloor\frac{3 k}{k-2}\right\rfloor\right)
$$

\section{Proof:}

By Lemma $1, n\left(\alpha^{\mathrm{j}}-\alpha^{\mathrm{p}}\right) \leq m-1$, iff $n\left[f\left(\frac{k-1}{k}\right)-f\left(\frac{1}{2}\right)\right] \leq m-1$, or 


$$
\frac{m-1}{n} \geq\left[f\left(\frac{k-1}{k}\right)-f\left(\frac{1}{2}\right)\right]
$$

By definition of the function $f,\left(\frac{k-1}{k}+\frac{1}{n}\right)$ and $\left(\frac{k-1}{k}+\frac{1}{n k}\right)$ are, respectively, upper and lower bounds of $f\left(\frac{k-1}{k}\right)$. Similarly, $\left(\frac{1}{2}+\frac{1}{n}\right)$ and $\left(\frac{1}{2}+\frac{1}{2 n}\right)$ are, respectively, upper and lower bounds of $f\left(\frac{1}{2}\right)$. Hence,

(A5) $f\left(\frac{k-1}{k}\right)-f\left(\frac{1}{2}\right) \leq\left(\frac{k-1}{k}+\frac{1}{n}\right)-\left(\frac{1}{2}+\frac{1}{2 n}\right)=\frac{k-2}{2 k}+\frac{1}{2 n}$

and

(A6) $f\left(\frac{k-1}{k}\right)-f\left(\frac{1}{2}\right) \geq\left(\left(\frac{k-1}{k}+\frac{1}{n k}\right)-\left(\frac{1}{2}+\frac{1}{n}\right)=\frac{k-2}{2 k}+\frac{1-k}{n k}\right.$

By (A6), a necessary condition for (A4) is:

$$
\frac{m-1}{n} \geq \frac{k-2}{2 k}+\frac{1-k}{n k}
$$

By (A5), a sufficient condition for (A4) is:

$$
\frac{m-1}{n} \geq \frac{k-2}{2 k}+\frac{1}{2 n}
$$

Substituting $m=2$ and $m=3$ in (A7) and (A8) we obtain, respectively, (10) and (11). 